\title{
A Low-Noise Potentiostat for the Study of Small Amplitude Signals in Electrochemistry
}

\author{
R. W. Shideler* and U. Berfocci† \\ National Bureau of Standards, Washington, D.C. 20234
}

January 25, 1980

\begin{abstract}
A low-noise potentiostat, developed and built at NBS, is described. The instrument, which has built-in an ac current amplifier, is particularly suited for detecting small current fluctuations in the frequency range between 0.1 and $2000 \mathrm{~Hz}$. The noise in the dc control voltage is of the order of $2.5 \times 10^{-8} \mathrm{~V} / \sqrt{\mathrm{Hz}}$, and the instability of the ac amplifier corresponds to a signal of $3 \times 10^{-11} \mathrm{~A} / \sqrt{\mathrm{Hz}}$. The performance characteristics of the instrument are fully described as well as its use in impedance measurements by means of a swept frequency signal. The instrument can also be used as a galvanostat.
\end{abstract}

Key words: Electrochemical measurements; galvanostat; impedance measurements; noise; potentiostat.

\section{Introduction}

The ability to detect and measure small amplitude signals in electrochemistry can be very useful when there is a need to minimize the input signal in order to perturb as little as possible the electrode/electrolyte interface as well as to decrease unwanted effects such as the ohmic drop error, and when the aim of the research is to study the spontaneous fluctuations in current and potential, which are commonly referred to as electrochemical noise. This area of endeavor is receiving increasing attention in the field of electrochemistry [1] $]^{1}$, and it is being applied for the detection of corrosion [2]. One of the problems encountered in these studies is caused by the need of controlling the potential of the electrode without causing an unacceptably high level of interference from the noise generated by the potentiostat [3]. In order to improve its performance with respect to commercially available instruments, a low-noise potentiostat was designed and built, whose characteristics will be described in the following sections.

\section{Instrument Characteristics}

The low-noise potentiostat was designed and developed by the Special Analytical Instrumentation Group of the Center for Analytical Chemistry at the National Bureau of Standards.

-Center for Analytical Chemistry, National Measurement Laboratory.

† Center for Materials Science, National Measurement Laboratory.

${ }^{1}$ Figures in brackets indicate literature references at the end of this paper.
The design has exceptional low noise and stable low frequency performance as well as moderately wide bandwidth.

The characteristics of the potentiostat include:

a. Highly stable operation over a wide range of loads with up to $\pm 5 \mathrm{~mA}$ output.

b. A useful frequency range from dc to greater than 2 $\mathrm{kHz}$.

c. A precision low noise voltage reference source variable from $\pm 5 \mathrm{~V}$, having a stability of better than $10 \mu \mathrm{V}$ for periods greater than $1 \mathrm{hr}$.

d. A fast recovery, low-noise, ac current signal amplifier with a frequency response of between $5 \times 10^{-2}$ and $2 \times$ $10^{3} \mathrm{~Hz}$.

Additional features include an external input to allow small signal modulation of the internal voltage reference and the ability to be used as a constant current source or galvanostat. The circuit scheme is shown in figure 1.

\section{Design and Construction Criteria}

In order to achieve the characteristics desired, the design was chosen so that the working electrode was grounded, facilitating the shielding of stray signals. To reduce capacitative currents across the reference electrode, the lead shield was maintained at the same potential, effecting a guarded input circuit. The high impedance guarded input amplifier is followed by the potentiostat loop amplifier and an output current buffer. (Total noise referred to input is less than $3 \mu \mathrm{V}$ rms.) The frequency response of the circuit 


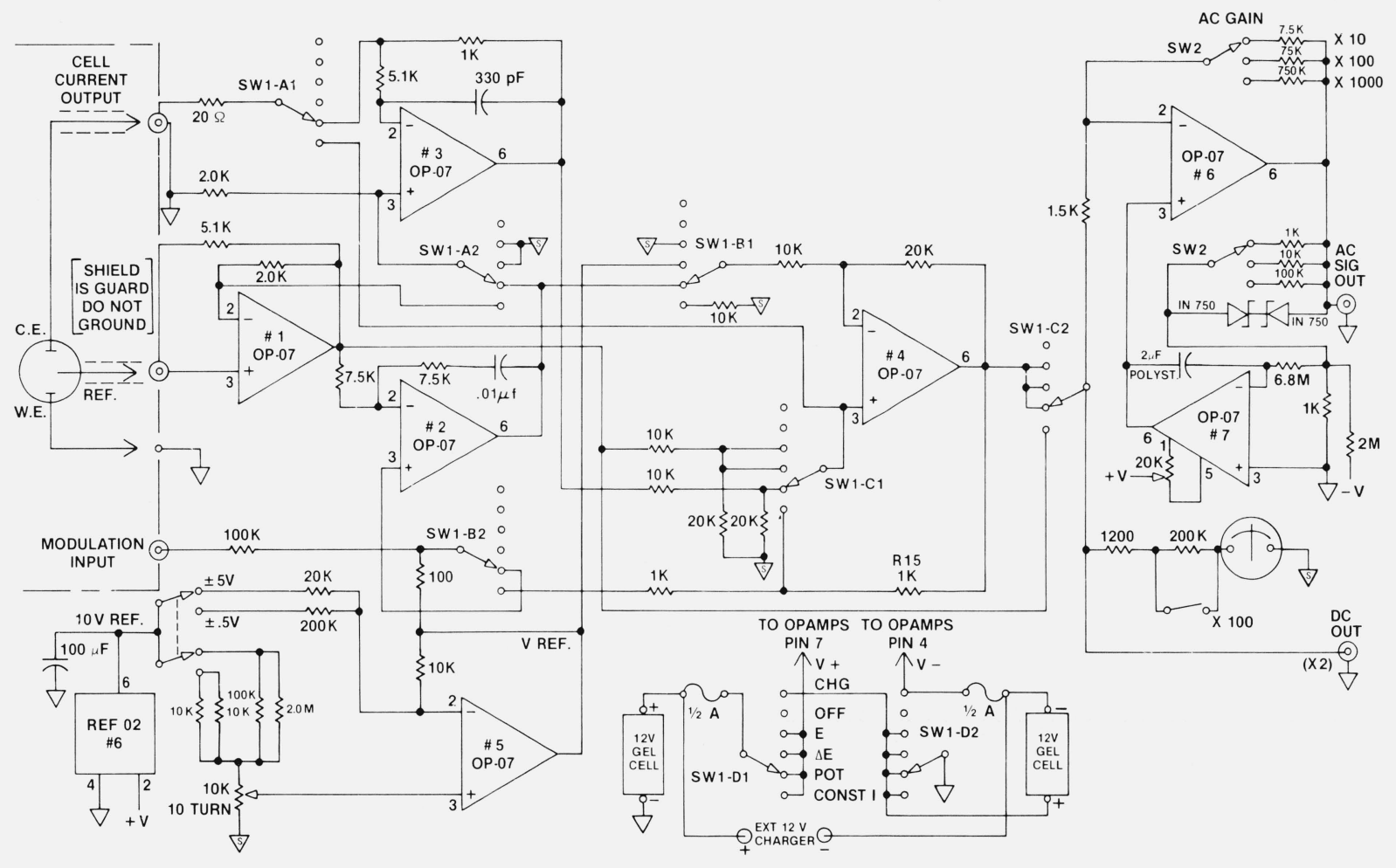

FIGURE 1. Schematic circuit of the potentiostat.

crosses unit gain at approximately $6 \mathrm{kHz}$. Immunity to oscillation for all values of cell $\mathrm{R}$ and $\mathrm{C}$ was secured by tailoring the frequency characteristics of the feedback amplifiers. No provision to compensate for cell parameters at high frequencies is included. A differential amplifier is used to extract the dc current signal from the potentiostat current driver. Signal output comes from an ac coupled amplifier having a very low frequency cutoff $\left(5 \times 10^{-2} \mathrm{~Hz}\right)$ and exhibits extremely low noise and prompt overload recovery. It has three gain settings up to a maximum gain of 1000. For ac components within the frequency range, an ultimate resolution as small as $3 \mathrm{nA}(3 \mathrm{mV}$ output) can be measured. A fast recovery circuit brings the amplifier into its linear range within a few seconds for any overload.

Power is supplied by a pair of rechargeable $12 \mathrm{~V}$ gelled lead acid batteries to assure a flat and constant discharge as well as complete freedom from ac pickup. All amplifier circuits make use of high performance operational amplifiers. The amplifier chosen has excellent noise and drift performance with long-term drift stability approaching that of chopper stabilized amplifiers.

A double box construction was used to reduce ambient thermal effects and improve immunity to electrical noise. Resistors used were glass-tin oxide, and all capacitors used in active circuit locations were of polystyrene foil construction. All loose wiring was mechanically secured by using dabs of silicone rubber sealant to reduce vibrations causing microphonics.

\section{Performance Characteristics}

The potentiostat is employed as a part of the circuit shown in a block diagram in figure 2. For testing purposes, the cell was substituted with a passive load, which was either a pure resistor or a resistor and a capacitor in parallel. With the switch in the upper position the fluctuations in the current were analyzed, while with the switch down, the noise voltage applied to the load was recorded. If desired, either a constant or a swept frequency signal of small amplitude was superimposed to the control voltage. All spectra shown in this work were recorded in four sections, over the $5,50,500$, and $5,000 \mathrm{~Hz}$ range. The spectrum analyzer has a frequency resolution of $1 / 200$ of the range, requiring a time of 40, 4, 0.4 and 0.04 s respectively for each spectrum. All recordings are averages over 64 spectra for the lowest range, and 256 or 512 spectra for the higher frequency ranges. All data given are rms values. 
Figure 3 shows the noise current for three values of the load resistance at zero current, as well as at $1 \mathrm{~mA}$ of dc current. Figure 4 shows the noise voltage $V_{N}$ for any resistive load at $I=0$ and $I=1 \mathrm{~mA}$, as well as the noise of the amplifier with the input shortcircuited. Subtracting this noise from the upper curve (at $I=0$ ) gives the dashed line, which represents the noise content $V_{C V}$ of the control voltage of the potentiostat in the frequency range of interest

$$
V_{C V}=\sqrt{V_{N}^{2}-V_{A}^{2}}
$$

This noise is largely independent of frequency above 1 $\mathrm{Hz}$ and is about $2.5 \times 10^{-8} \mathrm{~V} / \sqrt{\mathrm{Hz}}$. When applied to a re-

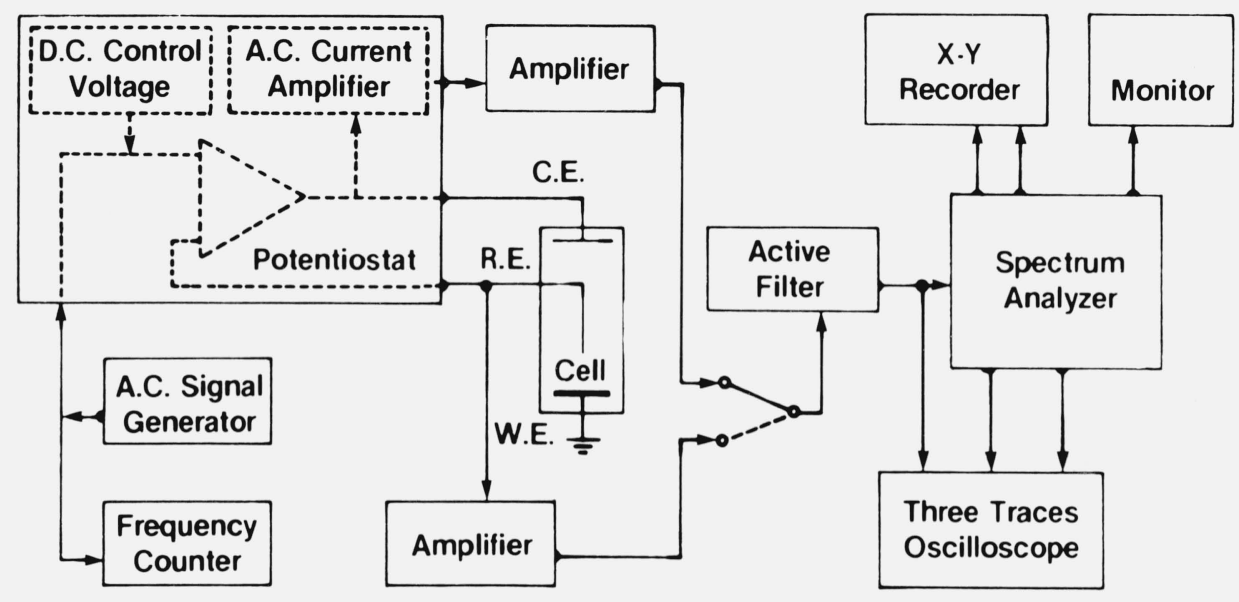

FIGURE 2. Experimental arrangement for recording current and voltage spectra.

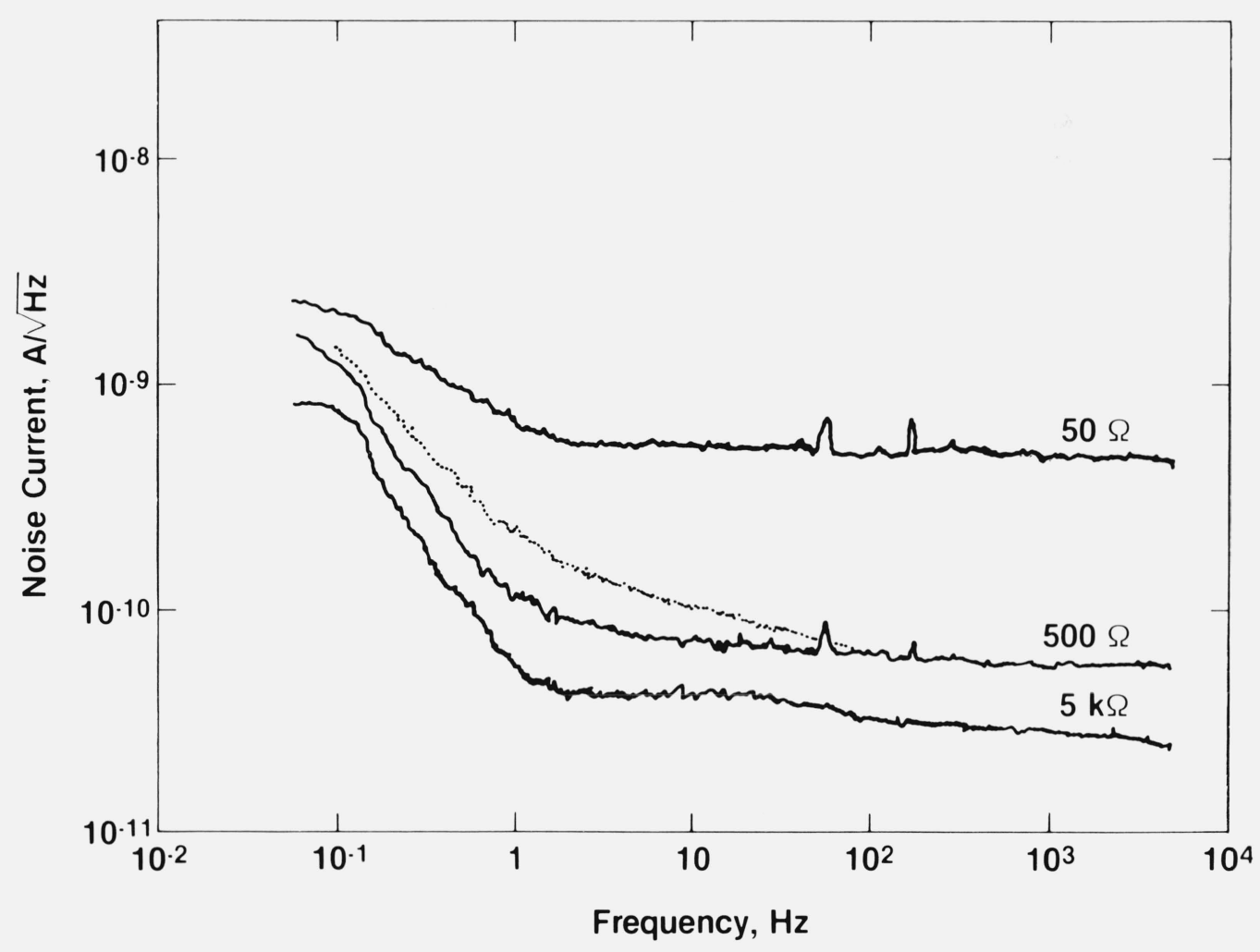

FIGURE 3. Current spectra recorded in potentiostatic conditions on different resistive loads. Solid lines: $I_{d c}=0$. Dotted line: $I_{d c}=1 \mathrm{~mA}$. 
sistive load this voltage signal generates the noise current $I_{N}$ shown in figure 3 . The noise current $I_{N}$ observed is the sum of the current $I_{c v}$ generated by the fluctuations in the control voltage and of a noise signal $I_{A}$ produced by the amplifying stage in the potentiostat.

$$
\begin{gathered}
I_{N}=\sqrt{I_{C V}^{2}+I_{A}^{2}}=\sqrt{\frac{V_{C V}^{2}}{R^{2}}+I_{A}^{2}}= \\
\sqrt{\frac{V_{N}^{2}-V_{A}^{2}}{R^{2}}+I_{A}^{2}}
\end{gathered}
$$

When the resistive load $R$ is sufficiently low $(<500 \Omega), I_{C V}$ predominates, as shown by the two upper curves in figure 3 . When $R$ is large, however, $I_{N} \sim I_{A}$, as shown by the lowest curve of figure $4 . I_{A}$ is also largely independent of frequency above $1 \mathrm{~Hz}$ and results to be about $3 \times 10^{-11} \mathrm{~A} / \sqrt{\mathrm{Hz}}$.

The potentiostat was also tested on a circuit formed by a $10 \Omega$ resistor in series with a parallel combination of a $5 \mathrm{~K} \Omega$ resistor and a $5 \mu \mathrm{F}$ capacitor (as shown in the inset in figure 5). In this case, the load impedance is frequency dependent. The voltage and current spectra are given in figure 5. Taking the ratio between these values at the same frequency should give the absolute value of the impedance $|Z|$.

$$
|Z|(\omega)=\frac{V_{C V}(\omega)}{I_{C V}(\omega)}=\sqrt{\frac{V_{N}^{2}-V_{A}^{2}}{I_{N}^{2}-I_{A}^{2}}}
$$

However, as shown before in the case of a resistive load, impedances greater than several hundred ohms are too large to give a current significantly above $I_{A}$ if one relies only on the control voltage noise. Because of the low intrinsic noise of the potentiostat, the addition of a very low amplitude swept frequency signal to the control voltage was sufficient for obtaining good impedance values. The voltage and current spectra obtained are shown in figure 6 , and the plot of $|Z|$ vs. frequency from which the value of the circuit components can be obtained, is shown in figure 7 . These measurements were obtained applying no more than a few tenths of a $\mathrm{mV}$ (in the lowest frequency range) and generating currents less than $100 \mathrm{nA}$. The possibility of measuring at low amplitudes can be an important advantage when, otherwise, distortions or irreversible changes in the electrochemical system could be produced.

\section{Performance as a Galvanostat}

As mentioned before, the instrument can be switched in a configuration where it supplies a constant dc current, up to $5 \mathrm{~mA}$, provided the total load does not exceed $5 \mathrm{~V}$. The ac

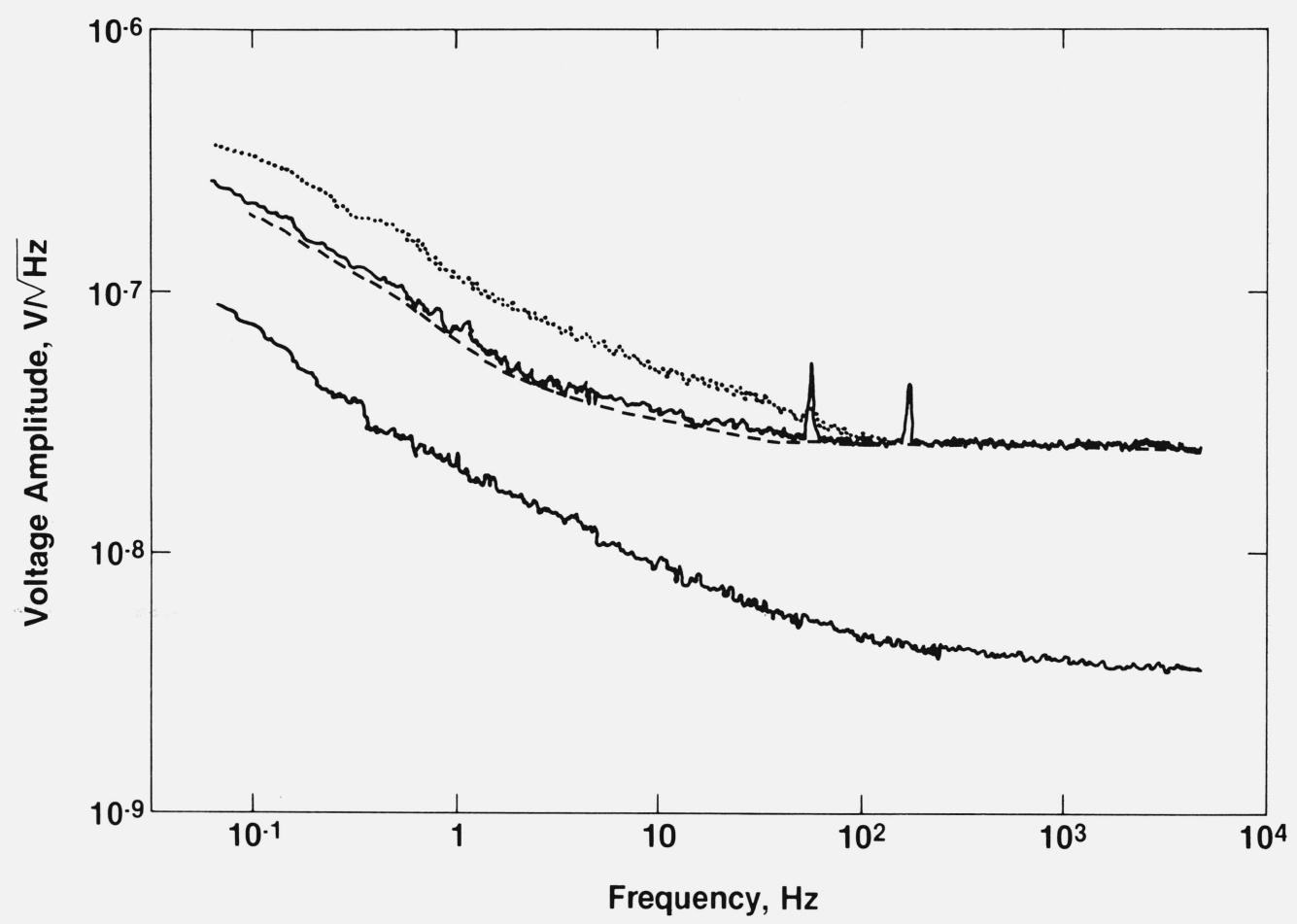

Figure 4. Voltage spectra.

Upper solid line: potentiostatic conditions, $I=0$. Dotted line: same, $I=1 \mathrm{~mA}$. Lower solid line: noise from amplifier with shortcircuited input. Dashed line: Difference between solid lines. 


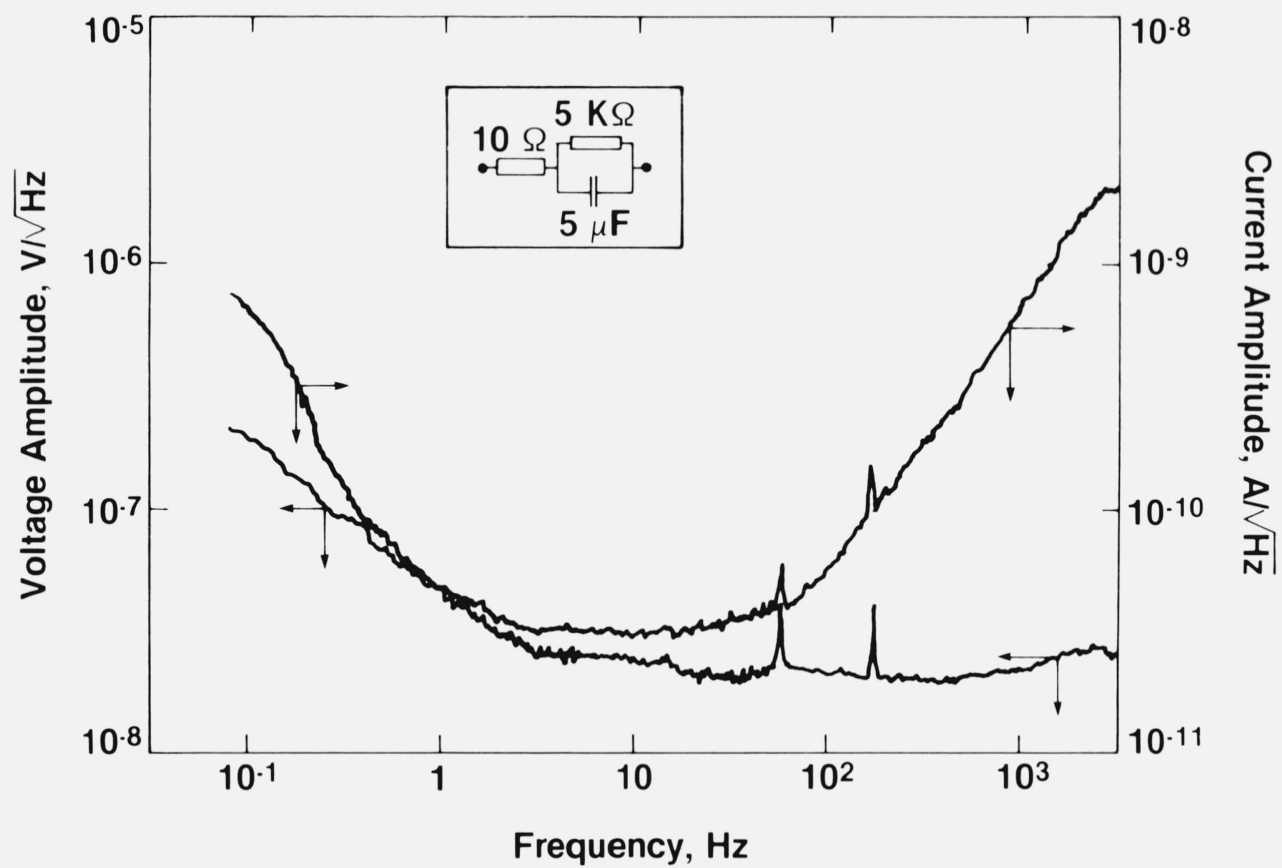

Figure 5. Current and voltage spectra recorded under potentiostatic conditions on circuit shown in inset.

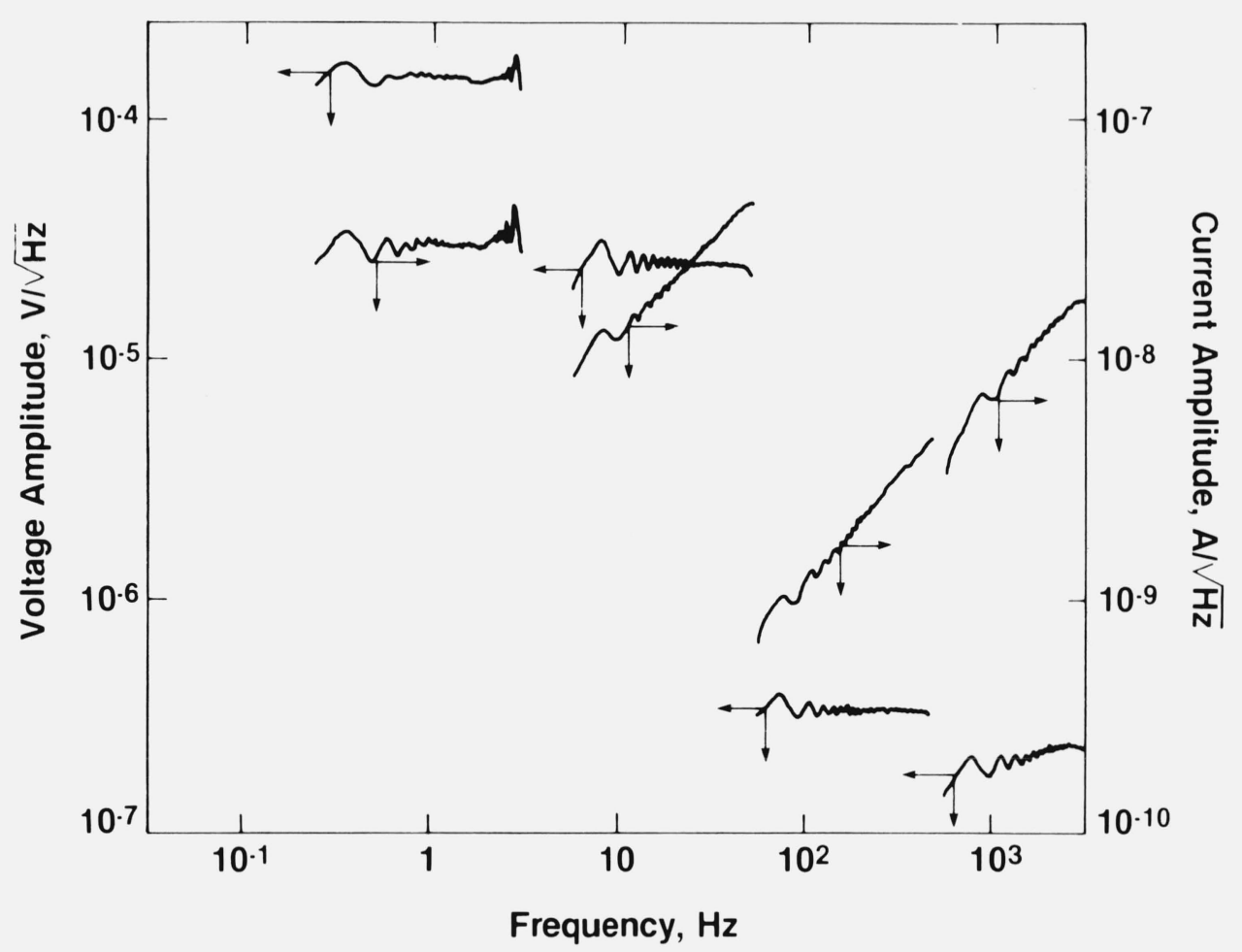

FIGURE 6. Current and voltage spectra recorded in potentiostatic conditions on the circuit shown in the inset in figure 5 .

Swept frequency signal superimposed to the dc control voltage. 


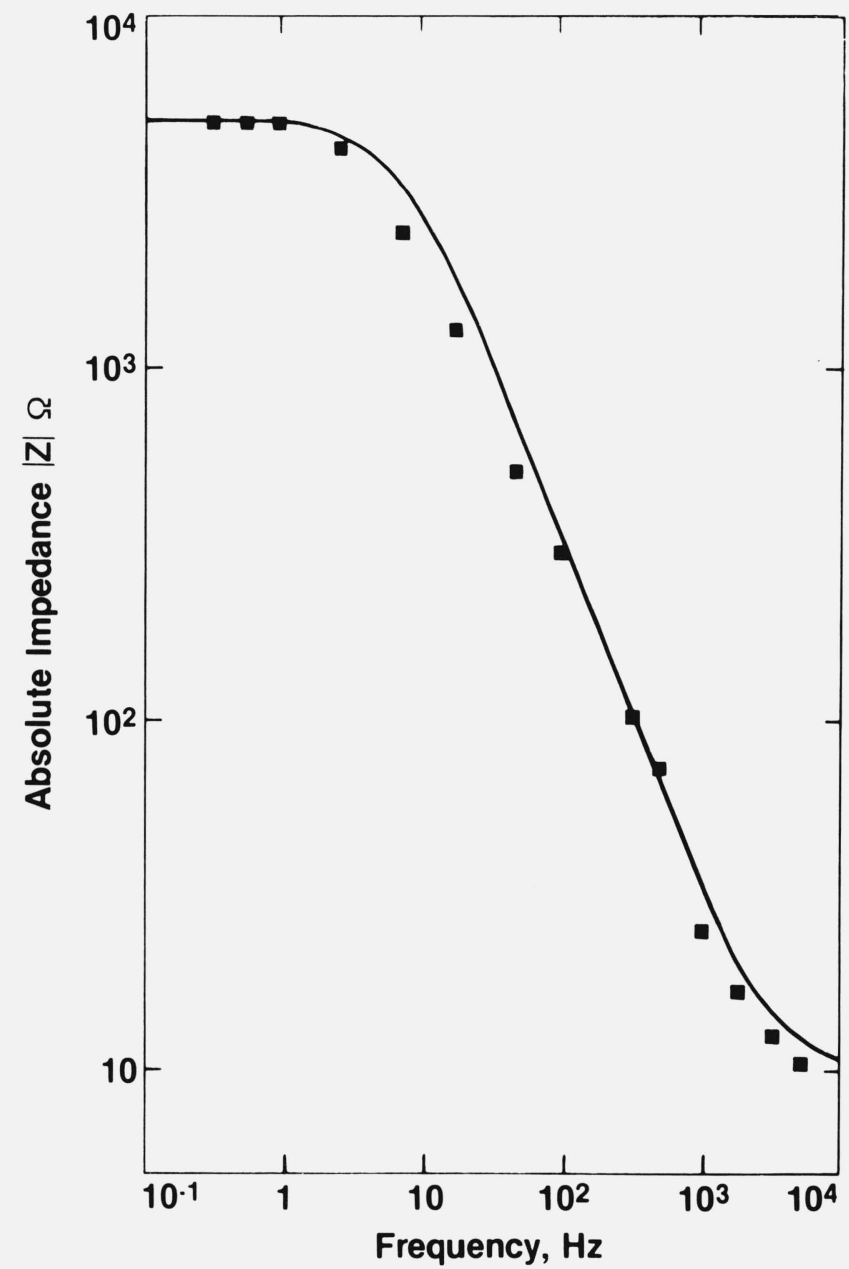

FIGURE 7. Absolute value of the impedance $|Z|$ vs. frequency for circuit shown in inset in figure 5.

Experimental points obtained from spectra in figure 6. Solid line: calculated values.

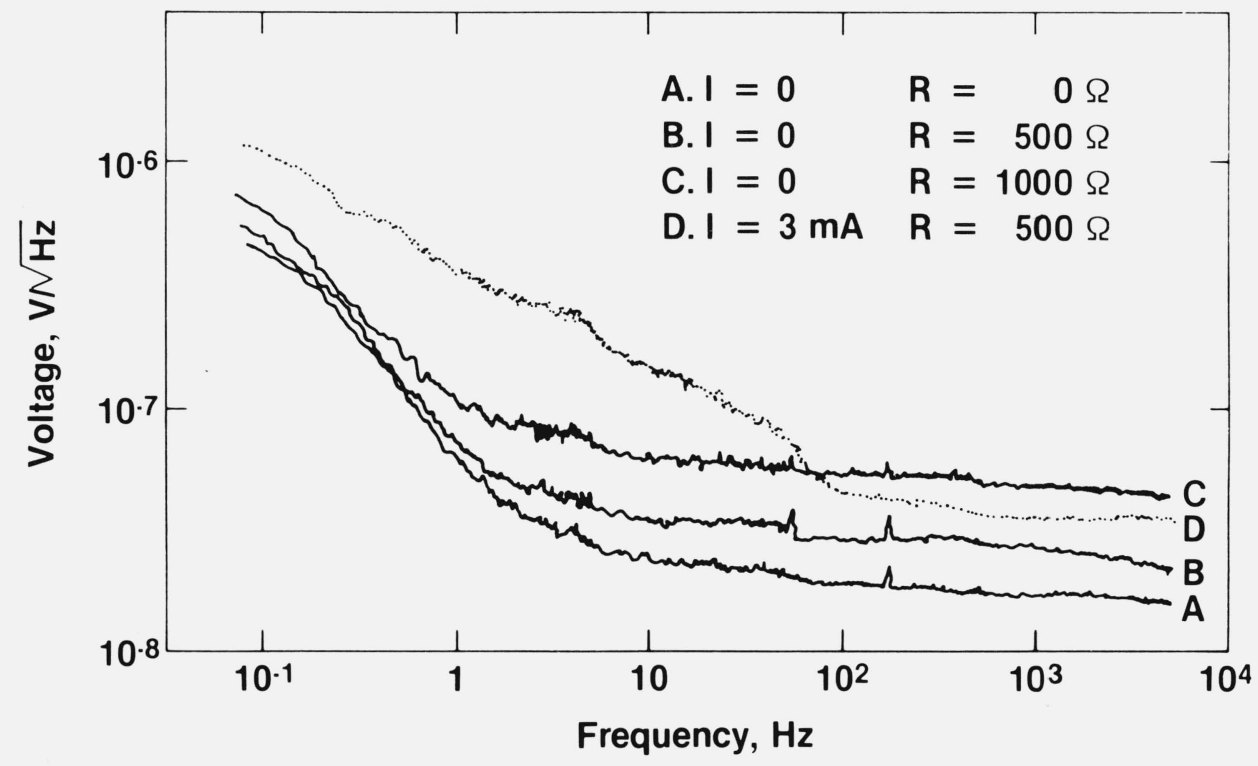

FIGURE 8. Voltage spectra recorded in galvanostatic conditions.

Curve A: $R=0, I=0$, Curve B: $R=500 \Omega, I=0$, Curve C: $R=1 \mathrm{~K} \Omega, I=0$, Curve D: $R=500 \Omega, I=3 \mathrm{~mA}$ 
content of the potential difference between working and reference electrode appears at the output of the ac amplifier.

Recordings taken with a resistor as load are shown in figure 8. The noise voltage, in a manner analogous to the potentiostatic case, is the sum of the noise generated by the instability of the control voltage $V_{C V}$, which in this configuration becomes a noise current through the load, and of the amplifier noise $V_{A}$.

$$
V_{N}=\sqrt{\frac{V_{c v}^{2}}{R_{1}^{2}} Z^{2}(\omega)+V^{2}}
$$

$R_{1}$ is the internal resistance $\left(R_{1}=1 \mathrm{~K} \Omega\right)$ on which $V_{c v}$ acts to determine the current, and $Z(\omega)$ is the load impedance, which in this case, is a resistance $R$. In curve $A$ of figure 8 , the resistance is zero, and therefore $V_{N}=V_{A}$. The noise in the control voltage can then be obtained from (4), and it is close to that obtained in the potentiostat configura- tion. Curve D shows some increase in noise when a dc current is being drawn.

\section{References}

[1] Tyagai, V. A., Elektrokhimiya, 3, 1331 (1967). Blanc, G., Gabrielli, C., Keddam, M., Electrochim. Acta, 20, 687 (1975).

Blanc, G., Epelboin, I., Gabrielli, C., Keddam, M., J. Electroanal. Chem., 62, 59 (1975).

Barker, G. C., J. Electroanal. Chem., 82, 145 (1977).

Gabrielli, C., Ksouri, M., Wiart, R., J. Electroanal. Chem., 86, 233 (1978).

[2] Iverson, W. P., J. Electrochem. Soc., 115, 617 (1968).

Okamoto, G., Sugita, T., Nishiyama, S., Tachibana, K., Boshoku Gijutsu, 23, 439, 445 (1974).

Blanc, G., Epelboin, I., Gabrielli, C., Keddam, M., J. Electroanal. Chem., 45, 97 (1977)

Bertocci, U., 7th International Congress on Metallic Corrosion, Rio de Janeiro, 1978, p. 2010. ABRACO (1979).

[3] Epelboin, I., Gabrielli, C., Keddam, M., Raillon, L., J. Electroanal. Chem., 91, 155 (1978). 
